\title{
THE ACTIVITY AND VARIABILITY OF THE SUN AND SUN-LIKE STARS. II. CONTEMPORANEOUS PHOTOMETRY AND SPECTROSCOPY OF BRIGHT SOLAR ANALOGS
}

\author{
Jeffrey C. Hall ${ }^{1}$, Gregory W. Henry ${ }^{2}$, G. Wesley Lockwood ${ }^{1}$, Brian A. SkifF ${ }^{1}$, and Steven H. SaAR ${ }^{3}$ \\ ${ }^{1}$ Lowell Observatory, 1400 West Mars Hill Road, Flagstaff, AZ 86001, USA; jch@lowell.edu \\ ${ }^{2}$ Center of Excellence in Information Systems, 3500 John A. Merritt Blvd., Box 9501, Nashville, TN 37209, USA \\ ${ }^{3}$ Harvard-Smithsonian Center for Astrophysics, 60 Garden St., MS 58, Cambridge, MA 02138, USA \\ Received 2009 April 6; accepted 2009 May 17; published 2009 June 11
}

\begin{abstract}
We present 14 years of contemporaneous photometric and spectroscopic observations of 28 solar analog stars, taken with the Tennessee State University Automatic Photometric Telescopes at Fairborn Observatory and the Solar-Stellar Spectrograph at Lowell Observatory. These are the best observed and most nearly Sun-like of the targets in our magnitude-limited $(V \leqslant 7.5)$ sample. The correlations between luminosity and activity reveal the expected inverse activity-brightness correlations for active stars. Strong direct correlations between activity and brightness are not prevalent for the less active solar age stars, but are precision limited. The Sun does not appear to have unusually low photometric variability when compared with the most Sun-like inactive solar analogs. We present evidence that the activity index $R_{\mathrm{HK}}^{\prime}$ is not a good discriminant of Maunder Minimum candidate stars. On the basis of a star that appears to have transitioned from a low-variability state to a cycling state, we investigate the regime in which stars might switch from faculae-dominated to spot-dominated variations.
\end{abstract}

Key words: stars: activity - Sun: activity

Online-only material: machine-readable and VO tables

\section{INTRODUCTION}

\subsection{Background}

The variability of Sun-like stars has been the subject of a number of long-term observing programs. For 37 years, Ca II $\mathrm{H} \& \mathrm{~K}$ measurements of a large set of stars were made at the Mount Wilson Observatory (MWO); essential compilations of the data and results were presented by Wilson (1978), Duncan et al. (1991), and Baliunas et al. (1995). The original set of 91 stars spanned spectral types F5 through M2, and, therefore, included only a limited set of "Sun-like stars" (defined by Cayrel de Strobel 1989 to encompass late F to mid G dwarfs or marginally evolved stars).

The MWO observations by themselves provided important insights into cool star activity and evolution, but they acquired a further dimension with the discovery that the solar "constant" varied directly with the Sun's activity cycle (e.g., Fröhlich \& Lean 1998; Willson 1997). Two decades of contemporaneous MWO H\&K measurements and Strömgren photometry of 34 Sun-like stars obtained at Lowell Observatory and Fairborn Observatory (Radick et al. 1998; Lockwood et al. 2007, hereafter L07) showed that this direct variation of brightness with activity was typical for older, lower activity solar analogs. Although the Sun appeared to be photometrically quiet relative to similar stars in this sample, the sample spanned a broad range of ages and properties, and the question of how stars very similar to the Sun (the so-called "solar analogs") might vary remained open. This question is important for understanding long-term solar luminosity variations during cycling periods as well as noncycling periods such as the Maunder Minimum of 1645-1715 (Eddy 1976)in accounting for those variations appropriately in terrestrial climate models, and, as more and increasingly less massive exoplanets are discovered, for the nature and evolution of exo-Earths. While recent work suggests that solar irradiance since 1700 has only increased by $\approx 1.0 \mathrm{~W} \mathrm{~m}^{-2}$ (Wang et al. 2005), the nature of stellar brightness variations in grand minima has relied so far upon snapshots of stellar ensembles using the Ca II H\&K proxy (Baliunas \& Jastrow 1990; Hall \& Lockwood 2004) rather than direct brightness measurements of good solar analogs. We (Hall \& Lockwood 2004) were unable to recover the Baliunas \& Jastrow result, and it has been argued that genuine Maunder Minimum candidates are few (Wright 2004; see however Saar 2006).

This paper continues the L07 investigation with combined spectroscopic and photometric measurements of bright solar analogs. Our instrument, the Solar-Stellar Spectrograph (SSS), can observe both the Sun and stars with the same instrumentation (Hall \& Lockwood 1995), and while our sample overlaps the MWO set to some extent, we have narrowed the great majority of our observations to stars with properties as close to the Sun's as possible within $V \lesssim 7.5$; we typically obtain $20-30$ observations of each of these stars per observing season. Meanwhile, synoptic photometry of these and many other Sun-like stars have been continued by Tennessee State University's Automatic Photometric Telescopes (APTs) at Fairborn Observatory in the Patagonia Mountains of southern Arizona (Henry 1999). The APTs obtain $\sim 60$ observations per season of each star in the sample.

\subsection{Solar Twins and 18 Scorpii}

A number of the high priority stars in the SSS sample are also on the APT program, including 18 Scorpii (HD 146233). We have recently compared the spectroscopy and photometry for this star for the period 1996-2006 (Hall et al. 2007), and we found that its brightness variations are very similar to the Sun's, with an amplitude of $0.09 \%$. This result raises a number of interesting questions. L07 concludes that while the Sun's $\mathrm{Ca} \mathrm{H} \& \mathrm{~K}$ activity cycle is vigorous relative to the cycles of their solar analogs, its photometric variability is quite low, even when a possible bias due to stellar inclination effects is removed. However, as noted above, the bulk of synoptic observations so far have been designed to elucidate the broad behavior of cool stars, many of which are quite different from the Sun. Even 18 
Sco itself is probably slightly younger than the Sun, rotates more rapidly, and has a dissimilar Li abundance (Porto de Mello \& da Silva 1997; Meléndez \& Ramírez 2007). These considerations and the 18 Sco photometric result force us to reconsider what we require of a "solar analog" and what observational requirements are in effect if we wish to use the stars as reliable proxies for past solar behavior.

Hardorp (1978) started the hunt for stellar analogs of the Sun, and after a decade of extensive discussion in the literature between Hardorp and his critics, Cayrel de Strobel (1989) wrote a comprehensive review of 109 solar analog candidates. According to her:

"A Sun-like star is a very broad class...of late F, early, middle, and sometimes, late $\mathrm{G}$ type dwarfs and subgiants."

"A solar analog is a Pop I dwarf with gross properties not very different from those of the Sun."

"A solar twin has fundamental physical parameters very similar, if not identical to those of the Sun."

By these admittedly qualitative descriptions, 18 Sco has so far been fairly called a solar twin (though, as noted above, better candidates are emerging as surveys move deeper). Its physical parameters apart from abundance match the Sun's to within $\approx 5 \%$ (Porto de Mello \& da Silva 1997; Soubiran \& Triaud 2004). However, its level of excess magnetic flux in Ca II H\&K appears higher than the Sun's (Hall et al. 2007), and although its activity cycle is of comparable amplitude, it is significantly shorter at $\approx 7$ yr. Also, as we will report below, the most recent data have substantially weakened the activity-brightness correlation reported by Hall et al. (2007). Despite this, its low photometric variability alone distinguishes it from the other stars in the L07 sample. Two factors may contribute to this.

First, there may be a precision bias. The total solar irradiance (TSI) variation of $\approx 0.1 \%$ requires millimagnitude photometric precision to detect, and, for a number of stars in the L07 sample, only upper limits were obtained.

Second, solar analogs as defined above may behave like the Sun in general but may fail to replicate its specific behavior over its activity cycle(s). Put more precisely, the size of the phase space in which stellar parameters must fall about the solar ones is sufficiently small that L07 observed no stars that are truly good proxies for solar variations.

In this paper, we address these issues by presenting the time series of the activity and brightness variations of 28 stars, continuing and extending the results of L07. Seven of these overlap the 32 star sample of that paper; the remainder includes all of the brightest solar analogs.

\section{OBSERVATIONS AND DATA ANALYSIS}

\subsection{Overview of Data}

In this paper, we expand our results for 18 Sco to include an additional 27 stars (and, for 18 Sco itself, an additional two seasons of observations). These stars are all on the high-priority observing lists of the SSS project and are those for which we also have good APT seasonal means. The sample, along with essential stellar parameters, appears in Table 1. Columns 1 and 2 give the HD number and the number of seasons for which we have both SSS H\&K and APT photometric seasonal means. In Columns 3 and 4 , we give the $V$ magnitude (Johnson et al. (1966) except where noted) and the $b-y$ color (from the catalogs by Olsen $(1993,1994)$ except where noted). In Column 5, we give the spectral type and in Column 6 the metal abundances,
Table 1

Target Stars and their Properties ${ }^{\mathrm{a}}$

\begin{tabular}{clllcrc}
\hline \hline HD & Seas & $V$ & $b-y$ & Spectral Type & {$[M / H]$} & Programs $^{\mathrm{f}}$ \\
\hline 1461 & 5 & 6.46 & 0.422 & G3 V & 0.16 & $\ldots$ \\
1835 & 12 & 6.39 & 0.412 & G2 V & 0.22 & LM \\
4307 & 8 & $6.16^{\mathrm{b}}$ & 0.394 & G0 V & -0.18 & $\cdots$ \\
10307 & 12 & 4.96 & 0.390 & G1 V & 0.10 & E \\
10700 & 9 & 3.50 & 0.438 & G8 V & -0.36 & M \\
20630 & 13 & 4.84 & 0.420 & G5 V & -0.10 & M \\
30495 & 12 & 5.51 & 0.403 & G1.5 V & -0.01 & M \\
35296 & 13 & $5.01^{\mathrm{c}}$ & $0.348^{\mathrm{e}}$ & F8 V & -0.12 & LM \\
38858 & 11 & 5.97 & 0.401 & G2 V & -0.18 & $\ldots$ \\
39587 & 13 & 4.41 & 0.376 & G0 V & 0.00 & LM \\
42807 & 10 & $6.44^{\mathrm{c}}$ & 0.418 & G5 V & -0.11 & $\cdots$ \\
43587 & 12 & 5.71 & 0.385 & G0 V & -0.08 & M \\
76151 & 13 & 6.00 & 0.416 & G2 V & -0.07 & M \\
82885 & 14 & 5.41 & 0.473 & G8 IV-V & 0.06 & LM \\
88986 & 7 & $6.46^{\mathrm{c}}$ & $0.396^{\mathrm{e}}$ & G2 V & -0.03 & $\cdots$ \\
90508 & 10 & 6.45 & 0.397 & G0 V & -0.23 & $\cdots$ \\
95128 & 4 & 5.05 & 0.392 & G1 V & 0.02 & E \\
97334 & 12 & $6.41^{\mathrm{d}}$ & $0.392^{\mathrm{e}}$ & G1 V & 0.08 & M \\
101501 & 13 & 5.34 & 0.445 & G8 V & -0.03 & M \\
115383 & 14 & 5.22 & 0.377 & G0 IV & 0.21 & LM \\
120136 & 14 & 4.50 & 0.320 & F7 IV-V & 0.25 & LM \\
140538 & 12 & $5.84^{\mathrm{c}}$ & 0.421 & G5 V & 0.06 & $\cdots$ \\
143761 & 9 & 5.40 & 0.393 & G0 + Va & -0.14 & LM \\
146233 & 12 & 5.49 & 0.409 & G2 Va & 0.03 & E \\
157214 & 6 & 5.39 & 0.402 & G0 V & -0.15 & $\cdots$ \\
168009 & 12 & $6.30^{\mathrm{c}}$ & 0.411 & G1 V & -0.02 & E \\
190406 & 11 & 5.80 & 0.384 & G0 V & 0.02 & $\cdots$ \\
197076 & 8 & $6.44^{\mathrm{c}}$ & 0.397 & G1 V & -0.09 & $\cdots$ \\
& & & & & & \\
\hline
\end{tabular}

Notes.

a Except where noted, $V$ magnitudes are from Johnson et al. (1966), and $b-y$ colors are from Olsen $(1993,1994)$. Metal abundances are taken from Valenti $\&$ Fischer (2005) and Gray et al. (2003).

b Carney (1978).

c Mermilliod (1986).

d Bidelman (1951).

e Crawford et al. (1966).

${ }^{\mathrm{f}} \mathrm{E}=$ ELODIE (Soubiran \& Triaud 2004), $\mathrm{M}=$ Mt. Wilson (Baliunas et al. 1995), L = Lockwood et al. (2007).

available for 23 of our targets in Valenti \& Fischer (2005) and for five others in Gray et al. (2003). Column 7 indicates other programs in which the star has been observed $(\mathrm{L}=\mathrm{L} 07$, $\mathrm{E}=$ ELODIE top ten solar analog (Soubiran \& Triaud 2004), $\mathrm{M}=$ MWO HK Project (Baliunas et al. 1995)).

Two observational quantities for each star comprise the essential data for this study. Spectroscopically, for each star we combine all SSS Ca II H\&K observations for a given observing season into a seasonal mean. We derive the excess flux $\Delta \mathcal{F}_{\mathrm{HK}}$ in $1 \AA$ rectangular bandpasses centered on the line cores, determined as described in detail by Hall et al. (2007), as well as the familiar $\log R_{\mathrm{HK}}^{\prime}$ and Mount Wilson $S$ indices. The "excess flux" is that portion of the total flux in the line cores arising from dynamo-related magnetic activity (obtained by removing estimates of photospheric flux and the "basal" chromospheric flux in the line cores). Photometrically, we use differential Strömgren $b$ and $y$ magnitudes to measure the seasonal mean brightness of our targets relative to low-variability comparison stars.

\subsection{Spectroscopic Data}

The HK series presented in this paper result from a reprocessing of our $\sim 30,000$ solar and stellar spectra following a seven 
Table 2

Activity-brightness Correlations

\begin{tabular}{|c|c|c|c|c|c|c|c|c|}
\hline HD & $\langle S\rangle$ & $\left\langle\log R_{\mathrm{HK}}^{\prime}\right\rangle$ & $\Delta \mathcal{F}_{\mathrm{HK}}(\odot=1)$ & $\sigma_{\star}$ & $\sigma_{c}$ & $\sigma_{f}$ & Var & Corr. \\
\hline Sun & 0.171 & -4.96 & 1.00 & $\ldots$ & $\ldots$ & $\ldots$ & $\ldots$ & $\ldots$ \\
\hline 1461 & 0.161 & -5.00 & 0.39 & 0.0003 & 0.0001 & 0.0003 & $\mathrm{v}$ & $52 /-$ \\
\hline 1835 & 0.350 & -4.43 & 7.61 & 0.0073 & 0.0013 & 0.0072 & $\mathrm{~V}+$ & $79 /-$ \\
\hline 4307 & 0.146 & -5.09 & 0.86 & 0.0005 & 0.0004 & 0.0004 & $\mathrm{C}$ & $21 /-$ \\
\hline 10307 & 0.158 & -5.00 & 0.71 & 0.0003 & 0.0004 & $\mathrm{u}$ & Vs & $83 /-$ \\
\hline 10700 & 0.159 & -4.99 & 0.60 & 0.0011 & 0.0006 & 0.0010 & $\mathrm{~V}$ & $17 /-$ \\
\hline 20630 & 0.359 & -4.43 & 6.97 & 0.0096 & 0.0009 & 0.0096 & $\mathrm{~V}+$ & $99 / \mathrm{I}$ \\
\hline 30495 & 0.297 & -4.52 & 6.47 & 0.0061 & 0.0010 & 0.0060 & $\mathrm{~V}+$ & $96 / I$ \\
\hline 35296 & 0.314 & -4.43 & 14.99 & 0.0030 & 0.0007 & 0.0029 & $\mathrm{~V}+$ & $81 /-$ \\
\hline 38858 & 0.182 & -4.88 & 1.54 & 0.0003 & 0.0003 & 0.0001 & Vs & $21 /-$ \\
\hline 39587 & 0.326 & -4.43 & 11.00 & 0.0054 & 0.0007 & 0.0054 & $\mathrm{~V}+$ & $96 / I$ \\
\hline 42807 & 0.337 & -4.46 & 7.27 & 0.0062 & 0.0004 & 0.0062 & $\mathrm{~V}+$ & $68 /-$ \\
\hline 43587 & 0.165 & -4.96 & 1.07 & 0.0011 & 0.0005 & 0.0010 & V & $60 /-$ \\
\hline 76151 & 0.254 & -4.64 & 3.53 & 0.0010 & 0.0008 & 0.0007 & $\mathrm{~V}$ & $22 /-$ \\
\hline 82885 & 0.262 & -4.70 & 1.98 & 0.0109 & 0.0006 & 0.0109 & $\mathrm{~V}+$ & $99 / \mathrm{I}$ \\
\hline 88986 & 0.140 & -5.14 & 0.71 & 0.0004 & 0.0003 & 0.0002 & vs & $94 / d$ \\
\hline 90508 & 0.154 & -5.03 & 1.32 & 0.0011 & 0.0005 & 0.0010 & $\mathrm{~V}$ & $90 / d$ \\
\hline 95128 & 0.151 & -5.05 & 0.78 & 0.0009 & 0.0009 & 0.0006 & V & $35 /-$ \\
\hline 97334 & 0.329 & -4.44 & 9.85 & 0.0059 & 0.0016 & 0.0059 & $\mathrm{~V}+$ & $78 /-$ \\
\hline 101501 & 0.316 & -4.56 & 3.40 & 0.0043 & 0.0016 & 0.0041 & $\mathrm{~V}+$ & $90 / \mathrm{i}$ \\
\hline 115383 & 0.407 & -4.44 & 4.20 & 0.0052 & 0.0022 & 0.0050 & $\mathrm{~V}+$ & $99 / \mathrm{I}$ \\
\hline 120136 & 0.192 & -4.77 & 4.20 & 0.0019 & 0.0004 & 0.0019 & $\mathrm{~V}+$ & $46 /-$ \\
\hline 140538 & 0.206 & -4.80 & 1.78 & 0.0010 & 0.0006 & 0.0009 & V & $91 / \mathrm{i}$ \\
\hline 143761 & 0.145 & -5.10 & 0.84 & 0.0010 & 0.0006 & 0.0008 & $\mathrm{v}$ & $4 /-$ \\
\hline 146233 & 0.173 & -4.93 & 1.21 & 0.0003 & 0.0002 & 0.0002 & Vs & $53 /-$ \\
\hline 157214 & 0.157 & -5.01 & 1.09 & 0.0004 & 0.0001 & 0.0004 & vs & $92 / \mathrm{i}$ \\
\hline 168009 & 0.164 & -4.98 & 0.81 & 0.0005 & 0.0005 & 0.0003 & V & $49 /-$ \\
\hline 190406 & 0.200 & -4.79 & 2.82 & 0.0020 & 0.0007 & 0.0019 & $\mathrm{~V}+$ & $55 /-$ \\
\hline 197076 & 0.167 & -4.94 & 1.97 & 0.0006 & 0.0010 & $\mathrm{u}$ & $\mathrm{Cs}$ & $50 /-$ \\
\hline
\end{tabular}

month observing hiatus from 2008 January-July. The SSS was originally installed at the $1.1 \mathrm{~m}$ telescope at Lowell Observatory in 1988, and the aging electronics finally expired in 2008 January. We replaced the original TEK $512 \times 512$ CCDs with new Andor CCDs of nearly the same dimensions and pixel size, as well as new turnkey acquisition software. The higher sensitivity in the blue and drastically reduced read noise $\left(4 e^{-}\right.$versus 42 previously) allows us now to observe both at a higher cadence and at higher signal to noise $(\mathrm{S} / \mathrm{N})$. We have since rewritten our IDL-based reduction software to accommodate both the old and new spectral formats, extract and normalize all the data consistently, and stitch the pre-2008 and post-2008 time series together. The revised routines also use high $\mathrm{S} / \mathrm{N}$ spectra of all our targets obtained with the new cameras as references against which to ensure that there are no offsets or drifts over time in the older data and to avoid discontinuities at the boundary. A thorough discussion of our methods and data archive is in preparation, and explanations of the reduction procedures, the HK time series generation, and the full IDL software documentation are available online at the SSS Web site. ${ }^{4}$

\subsection{Photometric Data}

We acquired the photometric observations in this paper with the T4 0.75-m and the T8 0.8-m APTs at Fairborn Observatory. The T4 APT has a single-channel photometer based around an EMI 9124QB photomultiplier tube (PMT) and acquires $20 \mathrm{~s}$ integrations sequentially through Strömgren $b$ and $y$ filters. The T8 APT has a two-channel photometer that separates the $b$ and $y$ passbands with a dichroic filter and takes simultaneous

\footnotetext{
4 http://www.lowell.edu/users/jch/sss/index.php
}

30 s integrations with two separate 9124QB PMTs. On good nights, the typical precision of a single differential observation, as determined from pairs of constant stars, is $\sim 0.0015 \mathrm{mag}$ for $\mathrm{T} 4$ and $\sim 0.0011 \mathrm{mag}$ for T8. The T8 data are somewhat more precise than $\mathrm{T} 4$ primarily because of the longer integrations permitted by the efficiency of the two-channel photometer. We observe each target star on the APT program in a quartet with three ostensibly stable comparison stars in hopes that at least two of the comparison stars will be constant. Henry (1999) presents a detailed discussion of the automated telescopes and photometers, observing techniques, and data reduction procedures needed for long-term, high-precision photometry.

We use the two most stable comparison stars in each quartet to compute differential magnitudes in Strömgren $b$ and $y$ for each observation. We form the composite quantity $[b+y] / 2$ for each individual observation and combine all observations for a season to produce a seasonal mean. We therefore create two data series for each target star, one relative to each of the two comparison stars. To further reduce the uncertainty associated with variability of the comparison stars, we compute a weighted mean of these two series to produce the final photometric series.

\subsection{Results and Online Data}

Table 2 contains the results of this study. The first four columns give the target and its grand mean Mt. Wilson $S$, $\log R_{\mathrm{HK}}^{\prime}$, and excess flux (where 1.0 is the mean solar cycle 23 excess flux) derived from the SSS HK indices (Hall et al. 2007).

To determine the significance of observed photometric variations in the target star seasonal means, we calculate two quantities analogous to those presented by L07. The first of these is the 
Table 3

Seasonal Means

\begin{tabular}{cccccrrr}
\hline \hline HD & Season & $\langle S\rangle$ & $\left\langle\log R_{\mathrm{HK}}^{\prime}\right\rangle$ & $\begin{array}{c}\Delta \mathcal{F}_{\mathrm{HK}} \\
\left(\mathrm{erg} \mathrm{cm}^{-2} \mathrm{~s}^{-1}\right)\end{array}$ & $\sigma\left(\Delta \mathcal{F}_{\mathrm{HK}}\right)$ & $\begin{array}{r}(b+y) / 2 \\
(\mathrm{mag})\end{array}$ & $\sigma((b+y) / 2)$ \\
\hline 001461 & 1998.9 & 0.159 & -5.010 & $9.676 \mathrm{e} 4$ & $1.877 \mathrm{e} 4$ & -0.00047 & 0.00014 \\
001461 & 1999.9 & 0.162 & -5.000 & $1.147 \mathrm{e} 5$ & $2.259 \mathrm{e} 4$ & 0.00013 & 0.00017 \\
001461 & 2000.9 & 0.164 & -4.985 & $1.243 \mathrm{e} 5$ & $2.640 \mathrm{e} 4$ & -0.00020 & 0.00017 \\
001461 & 2001.8 & 0.164 & -4.982 & $1.259 \mathrm{e} 5$ & $9.645 \mathrm{e} 3$ & 0.00002 & 0.00017 \\
001461 & 2003.8 & 0.161 & -5.004 & $1.075 \mathrm{e} 5$ & $3.191 \mathrm{e} 4$ & 0.00018 & 0.00014 \\
001461 & 2005.8 & 0.166 & -4.977 & $1.364 \mathrm{e} 5$ & $2.485 \mathrm{e} 4$ & -0.00072 & 0.00014 \\
001461 & 2006.9 & 0.169 & -4.958 & $1.538 \mathrm{e} 5$ & $2.847 \mathrm{e} 4$ & -0.00002 & 0.00014 \\
001835 & 1994.8 & 0.319 & -4.490 & $1.791 \mathrm{e} 6$ & $7.788 \mathrm{e} 4$ & 0.00850 & 0.00152 \\
001835 & 1996.9 & 0.305 & -4.514 & $1.672 \mathrm{e} 6$ & $6.638 \mathrm{e} 4$ & -0.00309 & 0.00081 \\
001835 & 1997.8 & 0.329 & -4.470 & $1.872 \mathrm{e} 6$ & $7.635 \mathrm{e} 4$ & -0.00719 & 0.00074 \\
\hline
\end{tabular}

(This table is available in its entirety in machine-readable and Virtual Observatory (VO) forms in the online journal. A portion is shown here for guidance regarding its form and content.)

target's absolute ability $\sigma_{f}$, as shown in Columns 5-7. Let the mean variance of the target star relative to the two comparison stars be $\sigma_{\star}^{2}$ and the variance of the differential series of the two comparison stars be $\sigma_{c}^{2}$, then

$$
\sigma_{f}^{2}=\sigma_{\star}^{2}-\frac{1}{2} \sigma_{c}^{2}-\epsilon^{2}
$$

The final term $\epsilon$ represents the measurement precision of the seasonal mean differential magnitudes achieved by each APT. A separate $\epsilon$ is derived for each of the two telescopes from observed distributions of the variance of the yearly means of the best comparison star pair in each stellar quartet. We see a fuzzy lower bound of the measurement precision in each histogram at approximately the $2 \sigma$ location, from which we estimate $\epsilon=0.0003$ for T4 and $\epsilon=0.0002$ for T8. Column 7 lists the value of $\sigma_{f}$, computed from Equation (1), and represents the intrinsic (astrophysical) rms variability of each target star, corrected for comparison star variability and measurement uncertainty; A "u" in Column 7 denotes undetected variability in a target star where $\sigma_{f} \leqslant 0$.

In Column 8 , we list a second variability criterion, a classification derived from the correlation significance of the differential time series for the target versus each of its comparison stars as defined by L07: $\mathrm{V}=>99 \%$ significance, $\mathrm{v}=>95 \%$ significance, and $\mathrm{C}=$ constant. A designation of $\mathrm{V}+$ indicates photometric variability greater than $0.005 \mathrm{mag}$; we also adopt the designation "Vs" for variations comparable to the full amplitude of modern solar activity cycles ( $\leqslant 0.0015 \mathrm{mag}$ ). For the stars that overlap the L07 stars, we obtain the same result as L07 for all stars except HD 143761, which now receives a marginally variable ("v") classification rather than $\mathrm{C}$.

Finally, in Column 9, we classify the significance of the rank correlation between a star's activity and brightness. We give the significance as well as a general classification denoted by "D" and "I" for direct and inverse correlations, respectively. We use a capital letter where the significance exceeds $95 \%$ and a lower case letter where $90 \%<\rho<95 \%$.

To claim a meaningful correlation between activity and brightness, we require that (1) a star have at least a moderately significant $(>90 \%)$ correlation between $\mathrm{HK}$ and $(b+y) / 2$ and (2) that it is variable above the noise and comparison star variations (i.e., Column 7 of Table 2 is not "u"). As is evident in Table 2, there are some cases where the first criterion is fulfilled but the second is not; in these cases, we must regard the correlations as coincidences of small-number statistics.
The full data set for all the stars in this study is available as an online electronic table. Some of the data appear in Table 3. Columns 1 and 2 of this table give the HD number of the star and the midpoint of the photometric observing season. Columns 3-6 give the Mount Wilson $S, R_{\mathrm{HK}}^{\prime}$, and excess flux and its $1 \sigma$ error derived from our spectra via the methods detailed in Hall et al. (2007). Finally, Columns 7 and 8 give the seasonal mean $b+y / 2$ relative to the grand mean of all seasonal means for that star, along with the error.

\section{COMMENTS ON INDIVIDUAL STARS}

In this section, we discuss significant features in the time series of our targets. In the interest of brevity in the print version of this paper, we present figures of the spectroscopic and photometric time series for nine targets representative of the essential types of variation detected. Complete time series of these and all other targets on the SSS program are being made available on the SSS Web site at the URL referenced in Section 2.2.

\subsection{L07 Stars}

We have observed seven stars discussed by L07: HD 1835, $35296,39587,82885,115383,120136$, and 143761. They serve as useful controls for verifying the consistency of the data sets, particularly the cross calibration of the SSS to MWO time series. All but 143761 receive a variability classification of $\mathrm{V}+$ ( $>0.005$ magnitude variations at $>99 \%$ confidence), as they did in L07. Five show the expected inverse activity-brightness correlation, with HD 1835 and 35296 at $80 \%$ and the others at $>95 \%$. The subgiant HD 120136 ( $\tau$ Boo) continues to exhibit no significant correlation. As a subgiant, it is not of particular interest to this work, but it does serve as useful confirmation that where L07 saw little activity-brightness correlation, our HK time series recover the same answer. We thus gain confidence that both detections and nondetections between the photometry and the MWO versus SSS HK series are robust.

An especially lovely example of a near-perfect inverse correlation is HD 82885 (11 LMi), a former standard star that has been more variable in the past few decades (Skiff \& Lockwood 1986). The activity and brightness time series appear in Figure 1, with the SSS HK seasonal means in the upper panel and the photometric seasonal means in the lower panel. For the photometric data, we also show the comparison star data differential series as X's. The individual $b$ and $y$ series are also 


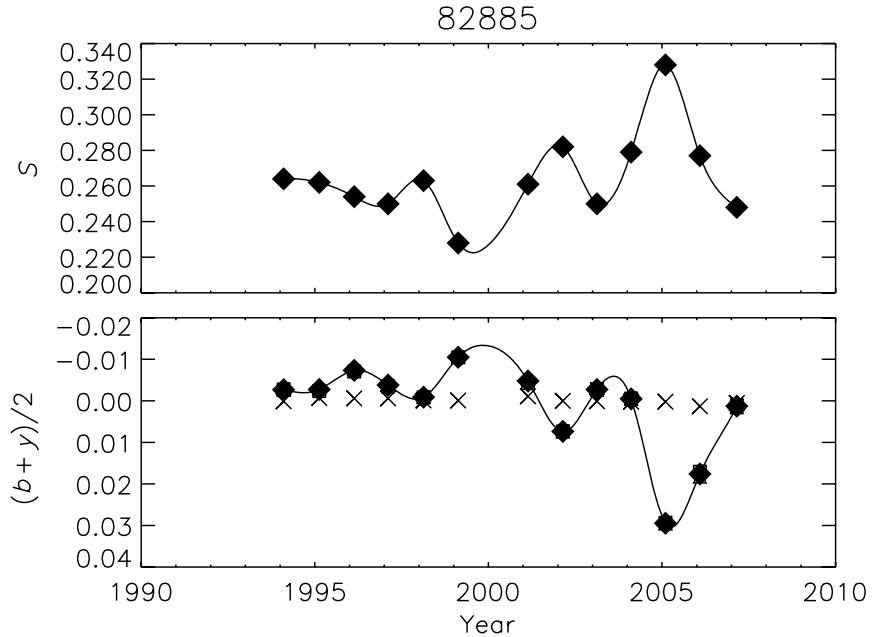

Figure 1. Ca II HK (top panel) and photometric (bottom panel) series for HD 82885. Diamonds are the seasonal means. The differential series of the comparison stars is shown by X's. The stars are well behaved and the individual points are hidden by the diamonds; this is not the case in time series with more variable comparison stars or lower amplitude program stars. Figures 2-9 are plotted identically to this one.

shown as open triangles and squares in this and subsequent figures; for HD 82885, these symbols are hidden by the target star symbols, indicative of an excellent pair of comparison stars (this is unhappily not always the case). Errors of the seasonal means are comparable to or smaller than the size of the plot symbols. All the $\mathrm{HK}+$ photometry time series in subsequent figures in this paper use this format.

The inactive star HD $143761\left(\rho \mathrm{CrB}, \mathrm{G} 0+\mathrm{Va},\left\langle R_{\mathrm{HK}}^{\prime}\right\rangle=\right.$ -5.10) appears in L07 as constant with a low-confidence inverse activity-brightness correlation. Baliunas et al. (1995) obtain $\langle S\rangle$ $=0.150$ and assign it a variability classification of "Long." We obtain variability "v" (weakly variable) with a positive absolute variability detection and no activity-brightness correlation. This star and HD 10700 ( $\tau$ Cet) are discussed by Judge \& Saar (2007) as potential Maunder Minimum candidates; we discuss them further in Section 4.

\subsection{Other Active Stars}

There are seven other stars in the sample for which we obtain a grand mean $R_{\mathrm{HK}}^{\prime}>-4.80$ : HD 20630, 30495, 42807, 76151, 97334, 101501, and 190406. All of these receive a V or $\mathrm{V}+$ variability classification and have weak to strong inverse activity-brightness correlations.

We obtain an inverse correlation at $>99 \%$ significance for HD 20630 ( $\kappa$ Cet; Figure 2). Baliunas et al. (1995) report a 5.6 yr period with cycle peaks in 1985 and 1991. This appears to continue in seasons of high activity in 1996 and 2002, but the most recent observations suggest continued low activity and higher luminosity through 2008. Two more seasons of observation should provide an interesting datum on possible cycle length variations in this star.

\subsection{HD 140538}

One of the most interesting targets in the sample is HD 140538 ( $\psi$ Ser). It is of intermediate activity $\left(\left\langle R_{\mathrm{HK}}^{\prime}\right\rangle=\right.$ -4.80) and is slightly cooler than the Sun (G5 V). The data appear in Figure 3. After four years of low variability (1997-2000), HD 140538 appears to have begun a vigorous four-year cycle, and our fall 2008 data following our CCD upgrade have con-

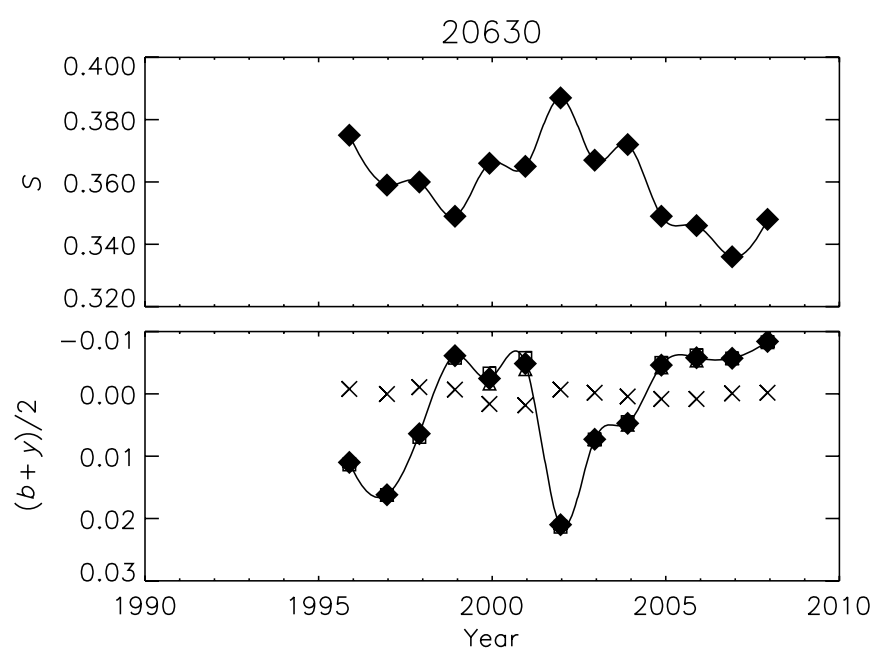

Figure 2. Time series for HD 20630.

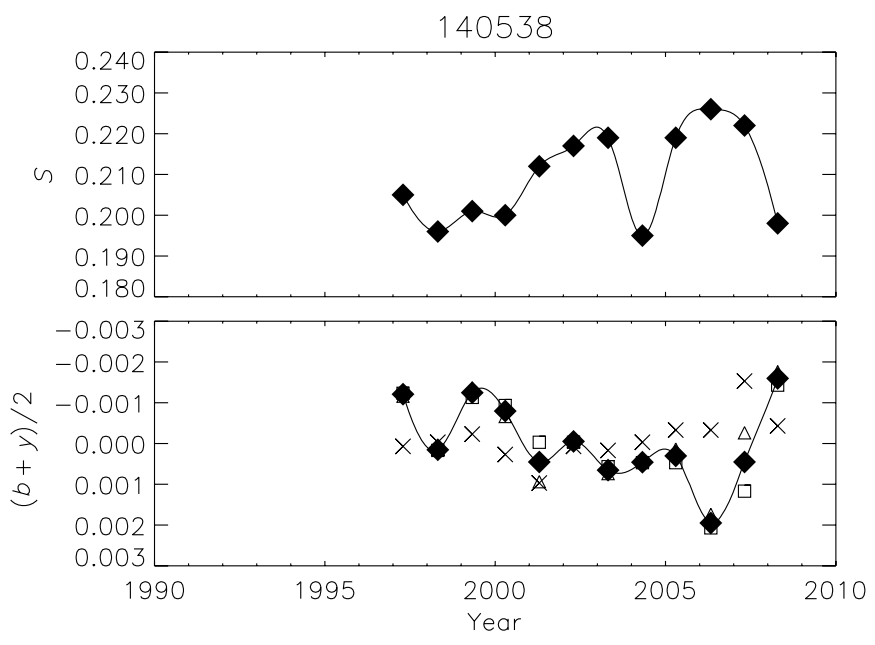

Figure 3. Time series for HD 140538 ( $\psi$ Ser).

firmed an apparent descent to a second minimum. It is demonstrably variable by the two criteria given in Section 2.4.

We detect a moderate inverse activity-brightness correlation for this star, but the $\mathrm{HK}$ and photometric time series present the opportunity for some interesting speculation. There is very little photometric variation during the 2000-2004 cycle, but an apparent inverse correlation over the 2004-2008 cycle. The four seasons prior to 2000 show a perfect direct correlation. Taking the observing seasons in groups of four, therefore, we have a tentative picture of a star that has "flipped" from direct brightness variations with sedate chromospheric activity, to a relatively flat period during which a strong cycle appeared, to inverse brightness variations over the subsequent cycle.

\subsection{HD 146233}

The solar twin HD 146233 (18 Sco; Figure 4) has been widely discussed (Porto de Mello \& da Silva 1997; Meléndez \& Ramírez 2007). It is not part of the L07 sample, and therefore was of particular interest when its rms photometric variability over more than a full $\sim 7$ year cycle was found to be the same as the Sun's, which placed 18 Sco close to the Sun's outlier position in L07's Figure 4; it also exhibited a fairly strong (>90\%) direct activity-brightness correlation (Hall et al. 2007).

Perhaps more attention to the cynic's adage about stopping while one is ahead would have been prudent, as we now must 


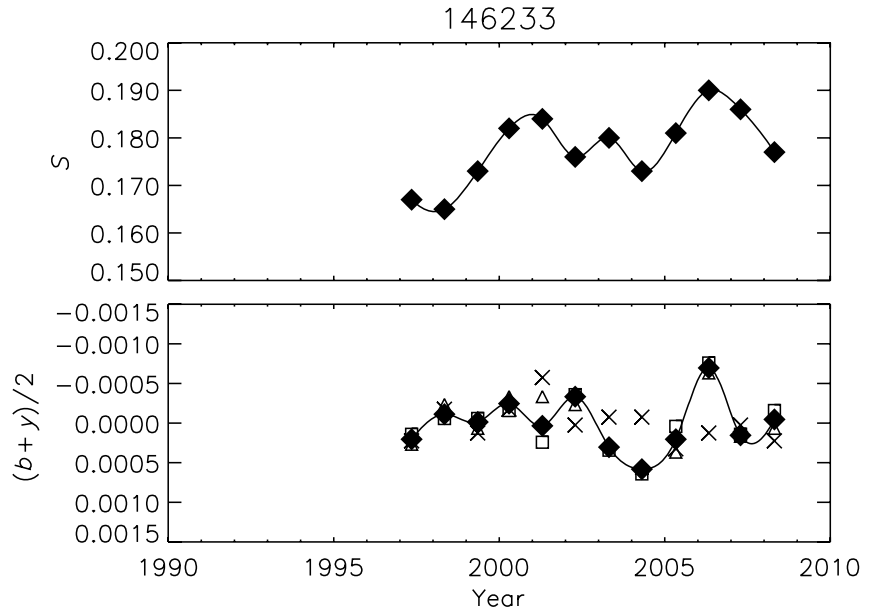

Figure 4. Time series for HD 146233 (18 Sco).

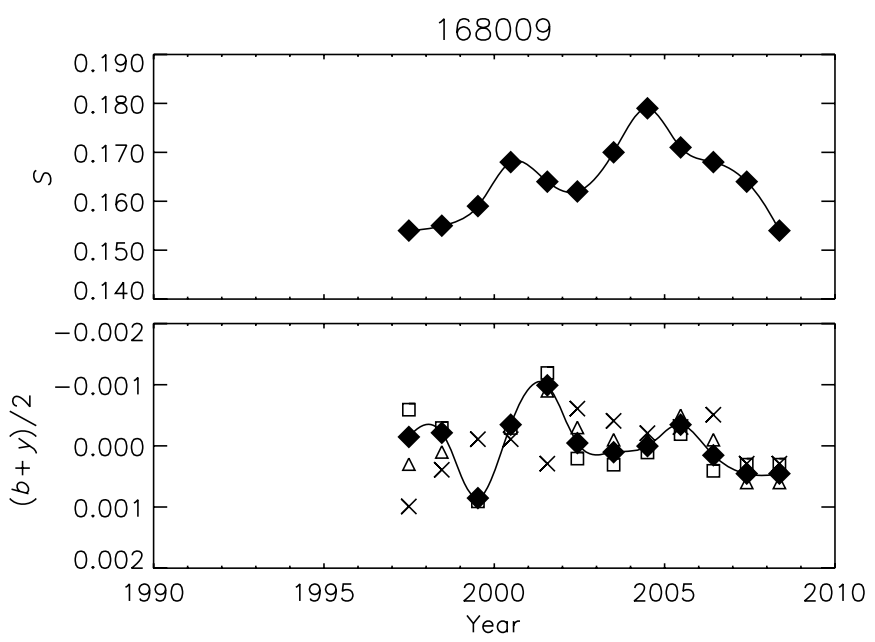

Figure 5. Time series for HD 168009.

report otherwise. First, the activity-brightness correlation of the most recent cycle disappeared in the two seasons subsequent to our earlier paper. The activity has declined as expected, but the photometric variability has not responded in kind; during the 2007 and 2008 observing seasons, 18 Sco's brightness was nearly constant at a level close to its 12 year mean, yielding a reduced variance of the overall time series. The comparison stars continue to be slightly variable as well. Second, as noted in Section 2, we have reprocessed our entire data set using the much higher S/N new spectra as references for the older spectra. While our ensemble results are unchanged, our stitching of the old and new CCD spectra via the newly acquired template observations shifted some of the older seasonal means, typically by $3 \%-10 \%$ and preferentially in the more inactive stars; irritatingly, the 18 Sco 2003 data are among them. This weakens our previously reported correlation; more significantly, the two most recent HK means are not correlated with the photometry, and we now find no significant correlation for the complete 12 year series. The amplitude of 18 Sco's activity and photometric variations, however, remain about the same as the Sun's.

\subsection{Additional ELODIE Solar Analogs}

In addition to $18 \mathrm{Sco}$, our sample includes three other stars on the ELODIE "top ten" solar analogs list (Soubiran \& Triaud 2004): HD 10307, HD 95128, and HD 168009. None of these show significant correlations, and HD 10307 is a photometric

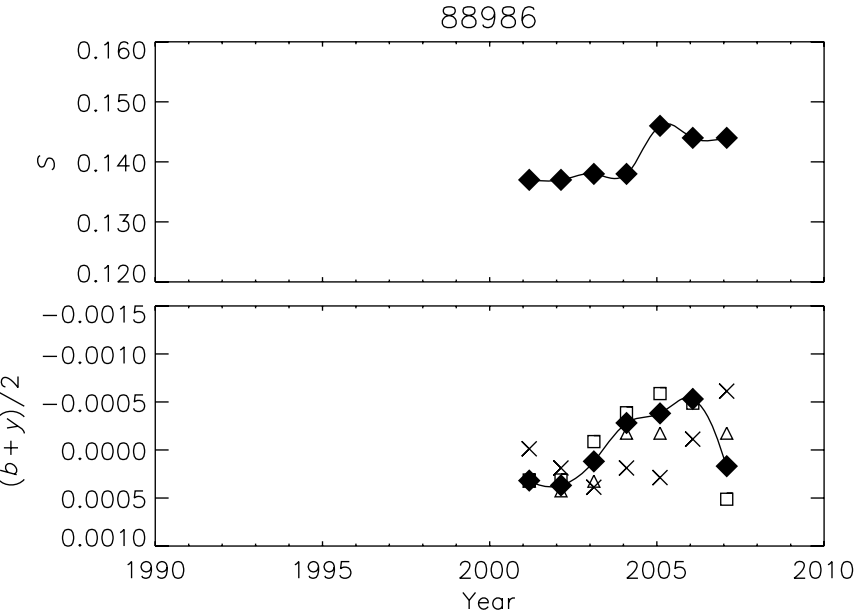

Figure 6. Time series for HD 88986.

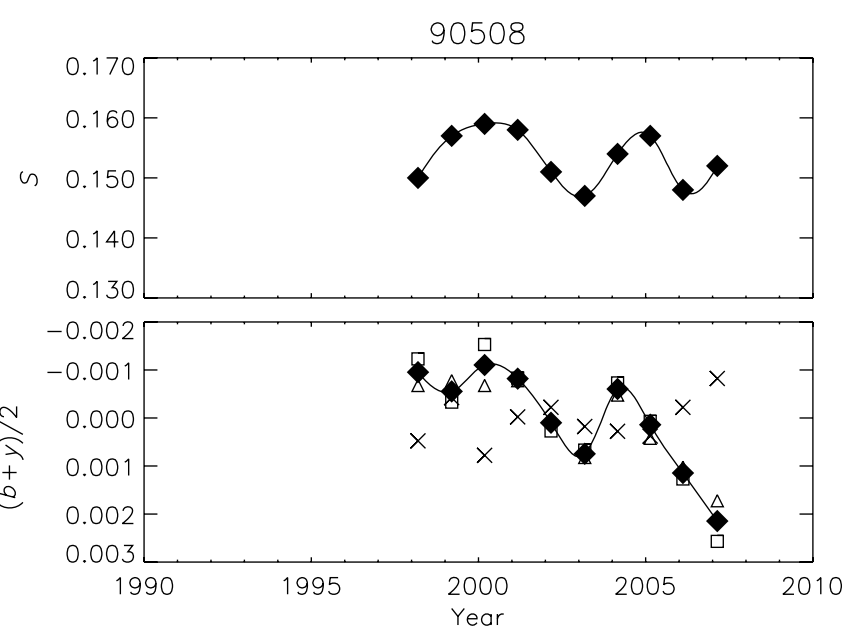

Figure 7. Time series for HD 90508.

nondetection. Interestingly, HD 168009 exhibits noticeable chromospheric variability at approximately solar $S$, but has been photometrically very quiet since 2002 (Figure 5). The time series is plagued throughout by variable comparison stars.

\subsection{Weak Direct Correlations}

In the 28-star sample, we observe only two stars with moderately significant (90\%-95\%) direct correlations: HD 88986 and HD 90508; the time series appear in Figures 6 and 7. The HD 88986 correlation appears spurious, since the time series covers only seven seasons and we do not detect photometric variability. Both these targets qualify as inactive stars by the $R_{\mathrm{HK}}^{\prime}$ criterion, with mean $\left\langle R_{\mathrm{HK}}^{\prime}\right\rangle$ of -5.03 for HD 90508 and -5.14 for HD 88986. We do not, however, find that HD 88986 is much less magnetically active than the Sun, since its excess flux $\Delta \mathcal{F}_{\mathrm{HK}}$ is comparable to the present solar minimum. The implications of this are discussed in Section 4.

\subsection{Inactive Stars}

The remaining seven stars are HD 1461, 4307, 10700, 38858, 43587, 157214, and 197076; all of them having solar or subsolar $R_{\mathrm{HK}}^{\prime}$. Of these, we detect photometric variability in only two (HD 10700 and 43587). One of the nondetections is HD 157214, which exhibits a moderate but probably spurious inverse activity-brightness correlation over a short time interval 


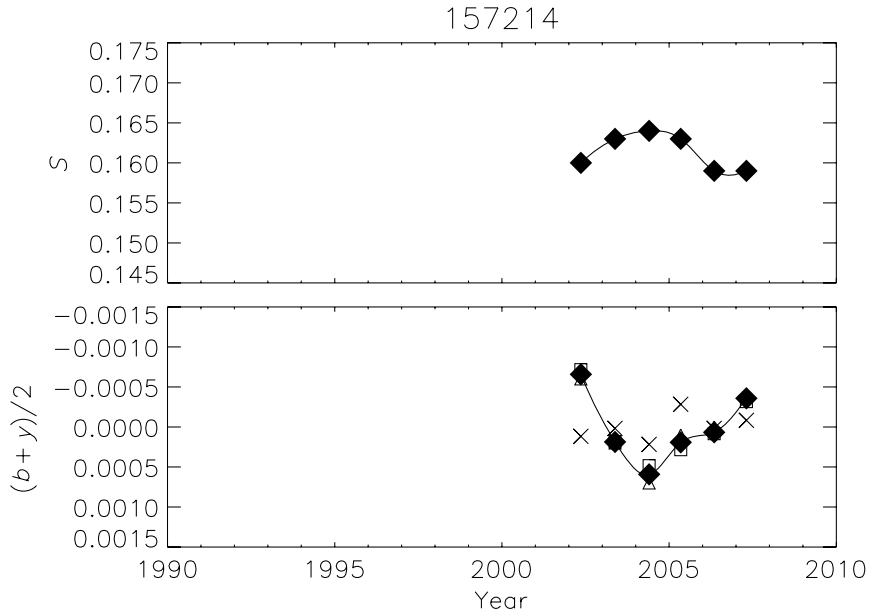

Figure 8. Time series for HD 157214.

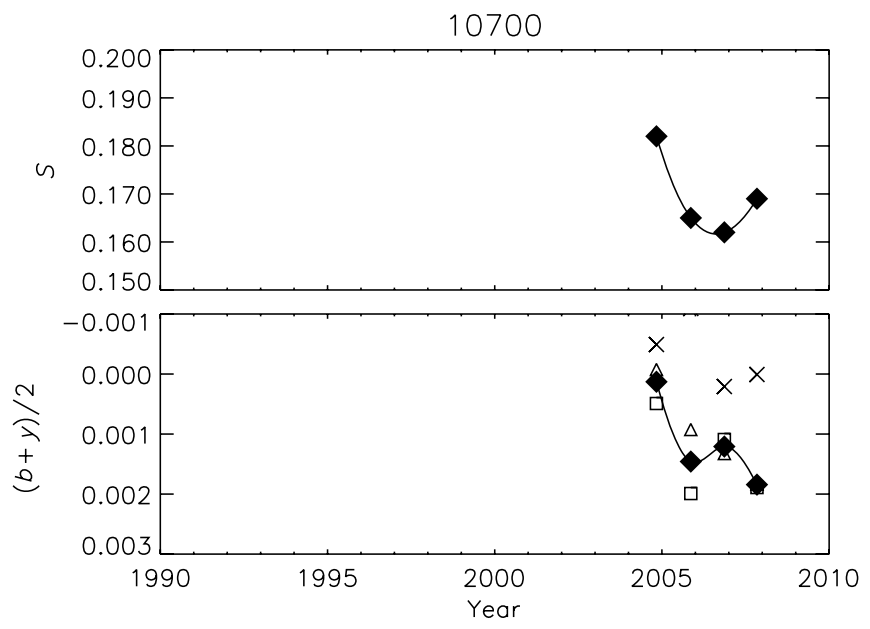

Figure 9. Time series for HD 10700.

of six seasons (Figure 8). Judge \& Saar (2007) have argued that HD 10700 ( $\tau$ Cet $)$ is a Maunder Minimum candidate and have found it to have near solar minimum magnetism on the basis of coronal observations, and we likewise find it to be variable in the spectroscopic and photometric time series (Figure 9). As with HD 140538, we see a shift from a period of strong activitybrightness correlation (inverse at $96 \%$ confidence from 1997 to 2005) to no correlation (2003-2008).

\section{DISCUSSION}

\subsection{Activity-Brightness Correlations and their Detectability}

In Figure 10, we plot the chromospheric flux fraction $\log R_{\mathrm{HK}}^{\prime}$ versus color for our 28 stars as well as the Sun; this figure is comparable to L07's Figure 8. The gray rectangle denotes the solar excursion in $\log R_{\mathrm{HK}}^{\prime}$ between 1994 and early 2009. Diamonds indicate stars with inversely correlated activity and brightness, while triangles indicate direct correlations. The two nondetections (i.e., those stars for which the target variability is less than the combined variations of the comparison stars plus noise, marked "u" in Table 2) are shown by open plot symbols. Large, medium, and small symbols indicate $>95 \%,>90 \%$, or nonsignificant correlations, respectively. The Sun is shown by a square.

The usual division of young, active stars with brightness varying inversely with activity and older, less active stars with

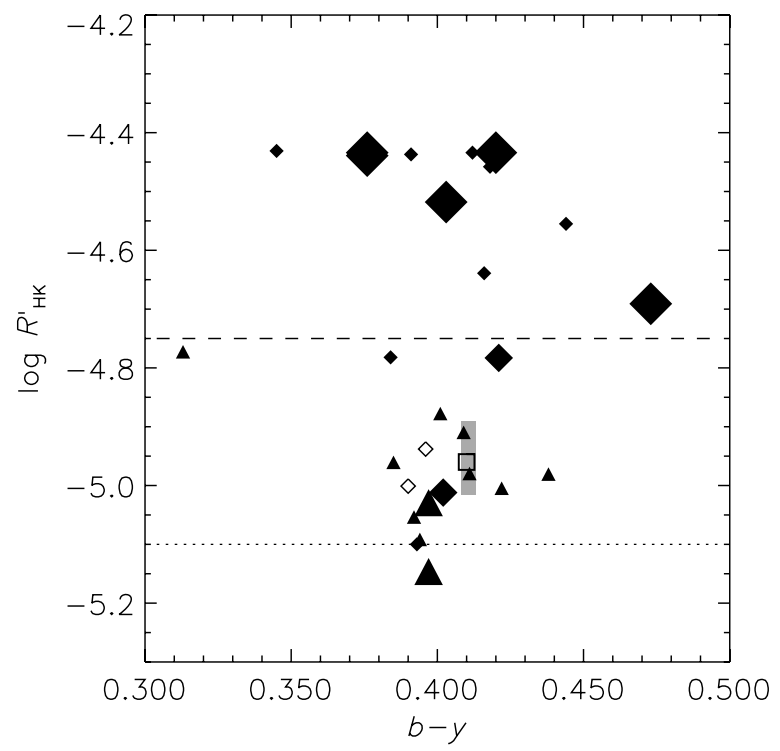

Figure 10. Color-activity diagram of our sample. Diamonds show inverse correlations and triangles show direct correlations. Open plot symbols are nondetections (i.e., no photometric variability apparent above the noise and the variability of the comparison stars), while filled symbols represent detections. Plot symbol sizes are proportional to the significance of the correlation. The Sun is shown by a square, and the gray bar shows the solar excursion in $R_{\mathrm{HK}}^{\prime}$ between 1994 and 2008. The dashed and dotted lines are the active-inactive and inactive-very inactive boundaries of Henry et al. (1996). The plot symbols in this figure are used consistently in Figures 11-16 as well.

brightness varying directly with activity is apparent, as discussed in detail by $\mathrm{L} 07$. The dashed line delimits a region $\left(\log R_{\mathrm{HK}}^{\prime}>\right.$ -4.75 ) where we find significant inverse correlations of activity and brightness; this matches the "active-inactive" boundary identified by Henry et al. (1996). Those authors also identified a "inactive-very inactive" boundary at $\log R_{\mathrm{HK}}^{\prime}<-5.1$, shown by the dotted line in Figure 10. In the present sample, we find strong $(>95 \%)$ inverse activity-brightness correlations for five stars, all significantly more active than the Sun. Direct (Sunlike) correlations are more elusive. We find no such correlations at $95 \%$ confidence, and only two (HD 88986 and 90508) at $>90 \%$.

The Sun has appeared in earlier work as an outlier on a plot of chromospheric activity versus long-term photometric variation (e.g., L07 Figure 7), prompting discussion of whether its sedate photometric variations arose from some sort of stellar uniqueness, from inclination effects (since the stars are presumably observed across a range of $\sin i$ while the Sun's variations are observed equatorially), or from a selection effect (the L07 sample is a subset of the MWO stars, which was selected to broadly sample F-K stars rather than to focus specifically on solar analogs). With the present sample, we are in a better position to examine this issue, and the results appear in Figure 11 (plot symbols here are the same as in Figure 10).

This figure is analogous to L07 Figure 7, and the dotted line shows the regression of the activity and photometric variability for the 32 stars in their sample. The dashed line is the regression of $\log R_{\mathrm{HK}}^{\prime}$ on the rms photometric variability for the 28 stars in our present sample (Table 2, Columns 3 and 5). Technically, the two nondetections are left-censored data, so we also ran regressions with these points removed and treated with a survival analysis (e.g., Isobe et al. 1986); with only two nondetections, there are insignificant changes to the regression in either case. Our data set and the L07 set are similar in number of targets 


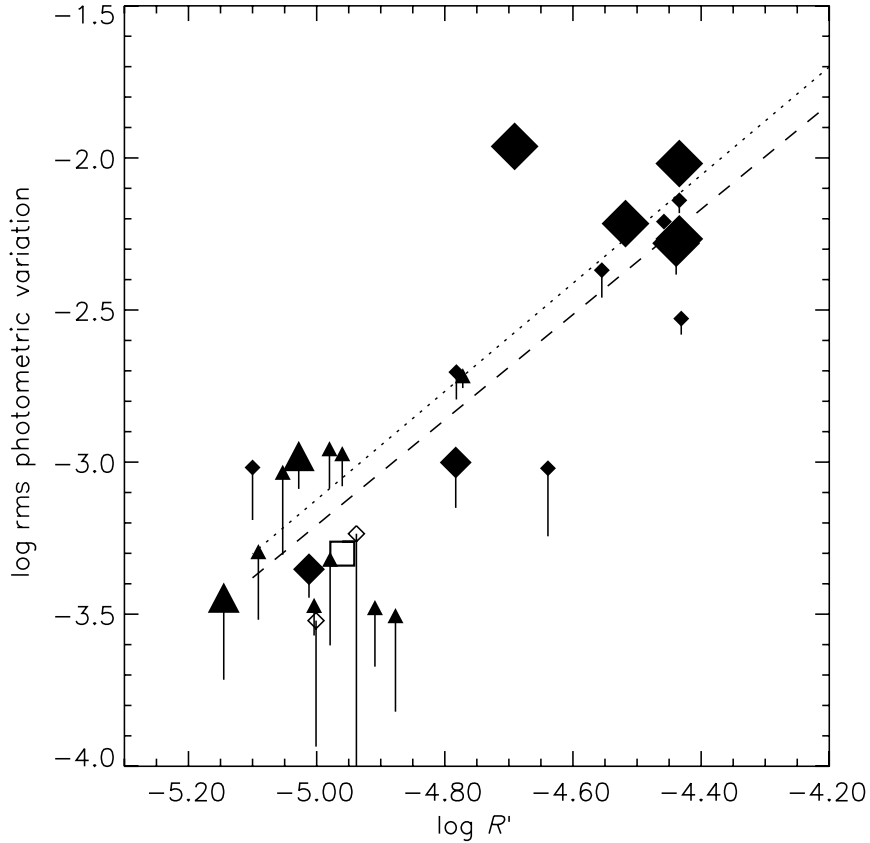

Figure 11. Activity vs. photometric variability of our sample. The Sun does not appear as an outlier among the variability envelope of inactive solar analogs. Drop lines from each plot symbol show where the point would move with perfectly stable comparison stars. The dotted line is the regression on the same scale as L07, while the dashed line is the regression obtained for the present sample.

and time series length, and we use directly comparable data (the same photometry, and consistent activity measurements (the $\log R_{\mathrm{HK}}^{\prime}$ and $S$ derived from the SSS data typically agree with MWO $S$ to $5 \%$ or less for inactive stars on both the surveys). The principal difference is our emphasis on solar analogs, and while the present regression has nearly the same slope as that of L07, it is shifted downward by about 0.1 dex in variability.

Figure 11 yields an interesting result. The detection threshold of our observations occurs at about 0.3 mmag (log rms variation $\sim-3.5$; see Equation (1)), corresponding to the precision limit of the APTs and about half the level of the rms cycle variability of the modern Sun. We observe five targets near this threshold of detectability, one of which is 18 Sco (lying at $\left.\log R_{\mathrm{HK}}^{\prime} \sim-4.90, \sigma((b+y) / 2) \sim-3.5\right)$. The Sun is therefore both no longer an outlier among the entire sample, and far from being unusually quiescent, it is above the median variability of the solar analogs with $\log R_{\mathrm{HK}}^{\prime}<-4.8$.

We also find five inactive stars with detected variability at $\sim 1$ mmag rms. Between this and the Sun is a gap of a factor of about 2 where we find no stars. Possibly this is a comparison star effect; drop lines on each symbol in Figure 11 show where the star would lie with perfectly behaved (i.e., $\sigma_{c} \approx \epsilon$ ) comparison stars. A larger sample is needed to determine if there is a real discontinuity between "Sun-like" and larger amplitude variations.

We can address two questions with these data. Straightforward is the issue of whether the Sun's low position in the Radick et al. (1998) and L07 activity-brightness variation plots is a selection effect: yes, it is. Less clear is whether the excursion of the Sun's activity cycle is unusual relative to those of genuine solar analogs and twins. In Figure 12, we show the mean $\log R_{\mathrm{HK}}^{\prime}$ for each of our targets versus its rms. The Sun lies at the top of the distribution of inactive stars, but several other targets are comparably variable, including $18 \mathrm{Sco}$, which has a cycle

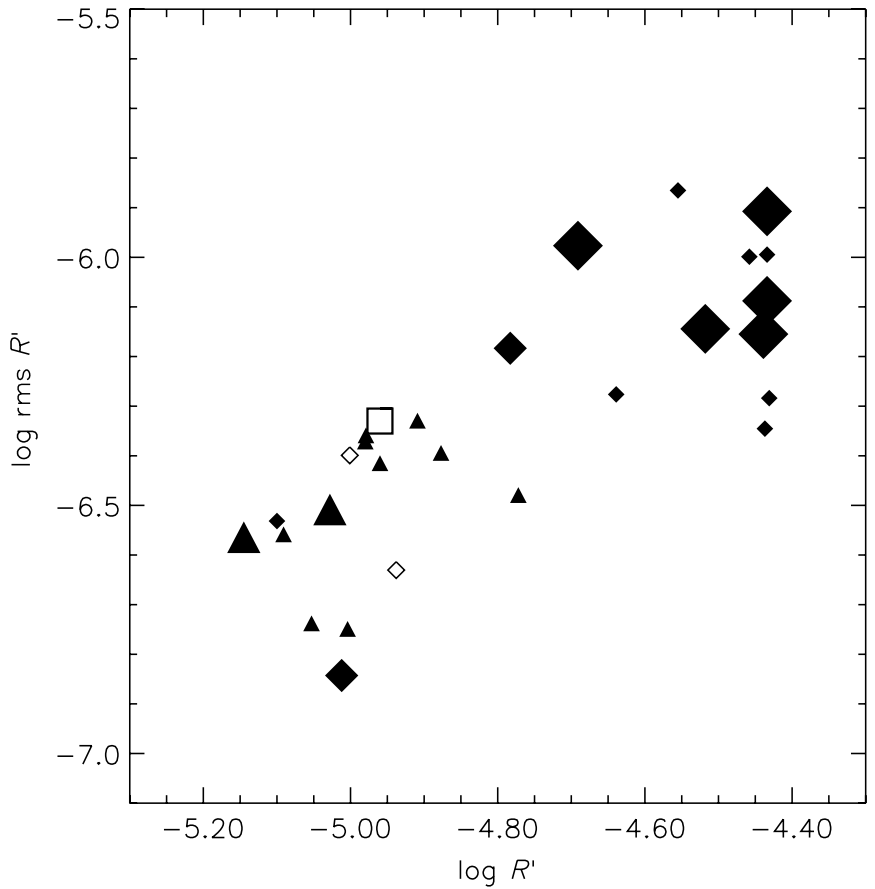

Figure 12. Relative chromospheric variability of the sample, showing the grand mean activity level for our HK time series vs. their rms. The Sun (open square) and 18 Sco (the filled triangle immediately to the Sun's right) are the most variable of the inactive stars in the sample.

variation of $\sigma\left(\log R_{\mathrm{HK}}^{\prime}\right)=0.043$ from 1994 to 2008 (comparable to 0.040 for the Sun over the same period). All we can say at this point is that the Sun and 18 Sco-the two inactive stars in the present sample with well defined cycles-(1) are the most chromospherically variable of the bright, inactive solar analogs and (2) vary with comparable amplitude.

\subsection{Assessment of $\log R_{\mathrm{HK}}^{\prime}, S$, and Excess Flux}

Although $\log R_{\mathrm{HK}}^{\prime}$ is a standard quantity in the literature, Rutten \& Schrijver (1987) found it less satisfactory than the surface flux density $\mathcal{F}$ for interpreting activity variations in cool stars and we (Hall et al. 2007) have discussed how we obtain the excess flux, defined as that part of the flux in a bandpass centered on the HK line cores arising from magnetic activity, and denoted $\Delta \mathcal{F}_{\mathrm{HK}}$. In Figure 13, we plot the same information as in Figure 10, except in terms of $\Delta \mathcal{F}_{\mathrm{HK}}$ rather than $\log R_{\mathrm{HK}}^{\prime}$. Again, the gray rectangle shows the range of solar excess flux we measured between 1994 and end 2008. The active-inactive star boundary translates from $\log R_{\mathrm{HK}}^{\prime} \approx-4.75$ to $\Delta \mathcal{F}_{\mathrm{HK}} \approx$ $5 \times 10^{5} \mathrm{erg} \mathrm{cm}^{-2} \mathrm{~s}^{-1}$, which is approximately twice the mean excess flux we measured in our solar spectra for Cycle 23.

However, Figure 13 shows that the $R_{\mathrm{HK}}^{\prime}$ picture becomes murkier below solar minimum (the bottom of the gray rectangle). Several stars that by $R_{\mathrm{HK}}^{\prime}$ standards are highly inactive are warmer than the Sun and therefore will lie higher on the flux axis, other things being equal, than on the $R_{\mathrm{HK}}^{\prime}$ axis. This effect is enhanced for inactive stars, where a smaller portion of the HK line core flux arises from dynamo-related processes than in more active stars with prominent emission reversals. While our sample appears to contain a number of "very inactive" $\left(\log R_{\mathrm{HK}}^{\prime}-5.1\right)$ stars lying quite close to the Sun on a color-magnitude diagram, they do not appear to be magnetically very different from the present excursion of the solar cycle. Two stars do appear to have low levels of magnetic flux: HD 1461, with $\Delta \mathcal{F}_{\mathrm{HK}} \sim 1 \times 10^{5}$ and 


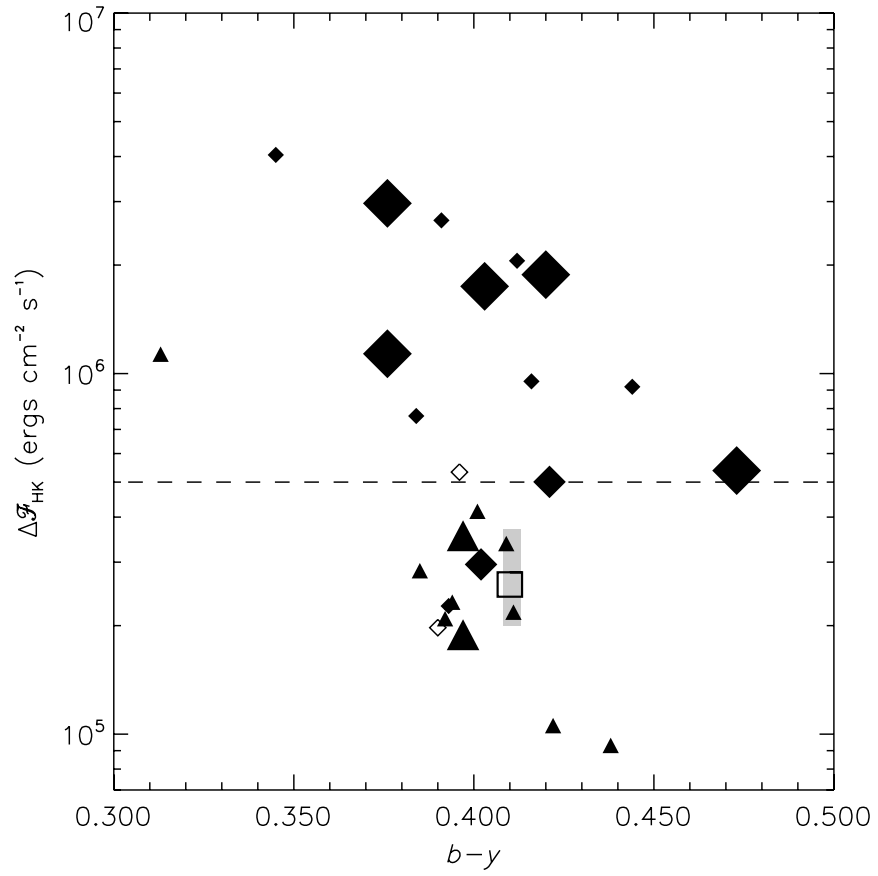

Figure 13. Comparable to Figure 10, except we express activity in terms of excess flux rather than $R_{\mathrm{HK}}^{\prime}$. Most stars significantly below the Sun in $R_{\mathrm{HK}}^{\prime}$ appear to be in magnetic states comparable to the modern solar excursion.

no detected photometric variability, and HD 10700, which we find to have $\Delta \mathcal{F}_{\mathrm{HK}} \sim 0.8$ of solar minimum and to be variable in both $\mathrm{HK}$ and $b+y$.

A second problem with $R_{\mathrm{HK}}^{\prime}$ emerges when we consider the spread in metal abundances for the stars in our sample (Table 1, Column 6). Saar (2006) noted that for dwarf stars (as defined by $\log g$ and $T_{\text {eff }}$ ) the minimum $R_{\mathrm{HK}}^{\prime}$ is a function of $[\mathrm{M} / \mathrm{H}]$, with metal-poor dwarfs having higher minimum $R_{\mathrm{HK}}^{\prime}$ than dwarfs of solar metallicity. This trend appears in our data set (see Figure 14), with the lowest $R_{\mathrm{HK}}^{\prime}$ stars tracing a lower bound of -4.95 at $[\mathrm{M} / \mathrm{H}] \sim-0.4$ and roughly -5.15 at $[\mathrm{M} / \mathrm{H}]=0$. We note that the two Maunder Minimum candidates of Judge \& Saar (2007), HD 143761, and HD 10700 lie on this boundary, and if this supposition is correct, we would submit HD 4307 and 88986 as additional candidates (although the somewhat low gravity of HD 4307 suggests it may be somewhat evolved (Valenti \& Fischer 2005), and the inferred excess magnetic flux of HD 88986 is comparable to that of the modern solar minimum). Maunder Minimum dwarfs may well be few (an issue examined by Wright 2004), but setting $R_{\mathrm{HK}}^{\prime} \leqslant-5.1$ as a criterion for Maunder minimumhood will exclude any even modestly metal-poor candidate dwarfs.

Similar issues apply to Mt. Wilson $S$; indeed they are more pronounced since $S$ (in contrast to $R_{\mathrm{HK}}^{\prime}$ ) is a "raw" measure of activity without any photospheric "background" correction. The distribution of $S$ versus color is similar to Figure 10, with the "inactive-very inactive" boundary appearing near the modern solar minimum of $S \sim 0.160$. However, the relative insensitivity of $S$ to differences in magnetic activity for low-activity stars results in a tight cluster of points near the Sun and above the theoretical minimum of $S \approx 0.14$ for Sun-like stars; this arises, of course, because most of the light in $S$ in these stars comes from the photosphere and quiet network. Judge \& Saar (2007) have made a similar argument, finding that $S$ is not a good indicator of surface magnetic fields in inactive stars.

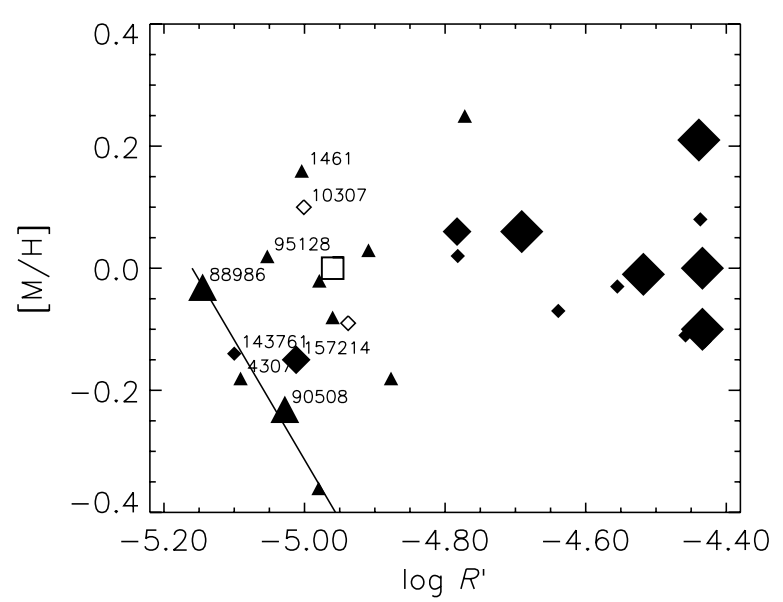

Figure 14. Dependence of $R_{\mathrm{HK}}^{\prime}$ on metallicity. The most inactive stars fall on a locus that increases with decreasing metallicity, consistent with Saar (2006). HD 4307, 10700, 88986, and 143761 emerge as the Maunder Minimum candidates of our sample, though two or possibly three of them would be excluded from consideration by an $R_{\mathrm{HK}}^{\prime}<-5.1$ criterion.

We therefore argue that even in this small sample, $\Delta \mathcal{F}_{\mathrm{HK}}$ is clearly the preferred unit, particularly in the regime of true solar twins and Maunder Minimum candidate stars. In an interesting recent paper, Schröder et al. (2009) have discussed the inadequacy of $\log R_{\mathrm{HK}}^{\prime}$ for subgiants and rapidly rotating stars. This is evident in Figures 10 and 13 as well; the two stars with significant inverse correlations at $b-y \approx 0.376$ are HD 39587 [GO V] and HD 115383 [G0 IV]. Their distinct activity in $\Delta \mathcal{F}_{\mathrm{HK}}$ is obscured in $\log R_{\mathrm{HK}}^{\prime}$, and this makes the point especially clearly since the background is less important fractionally in such a case. (Instead, perhaps the metallicity difference $([\mathrm{M} / \mathrm{H}]=0.0$ versus 0.21$)$ may be in play. $)$ The issues are not limited to rotation and evolution, though; in the key area of discerning differences in magnetic activity between otherwise similar stars, $\log R_{\mathrm{HK}}^{\prime}$ also obscures important differences. While $S$ and $R_{\mathrm{HK}}^{\prime}$ remain useful in a relative sense, as well as for facilitating direct comparison between new studies and a large body of literature, these are not ideal for attempting to identify genuine stellar analogs of solar grand minima. Other effects, such as gravity and metallicity, obscure their diagnostic value. Figures 10 and 11 are therefore presented as legacy items for comparison with the large body of work based on $\log R_{\mathrm{HK}}^{\prime}$, e.g., L07; at the same time, in terms of $\Delta \mathcal{F}_{\mathrm{HK}}$, the Sun is not found to be an outlier among its closest low-activity analogs.

\subsection{Correlation Regimes}

L07 have noted that the Sun lies close to the line at which the regression of brightness on activity switches from positive to negative (corresponding to a change from direct to inverse brightness-activity correlations). In Figure 15, we show the behavior of our present sample in this regard, but in terms of $\Delta \mathcal{F}_{\mathrm{HK}}$ rather than $R_{\mathrm{HK}}^{\prime}$. The full range of solar variability is shown by the gray bar. All stars within the solar excursion lie above the line (direct variations); all stars above it except HD 120136 are at or below it.

Perhaps a better hint of the detailed patterns of solar analog variation, however, is contained in Figure 16, in which we have calculated the correlation of activity in brightness in a four-year boxcar along the entire time series, for those stars which (1) were not photometric nondetections and (2) show an overall activity-brightness correlation significance of $\geqslant 90 \%$. We were 


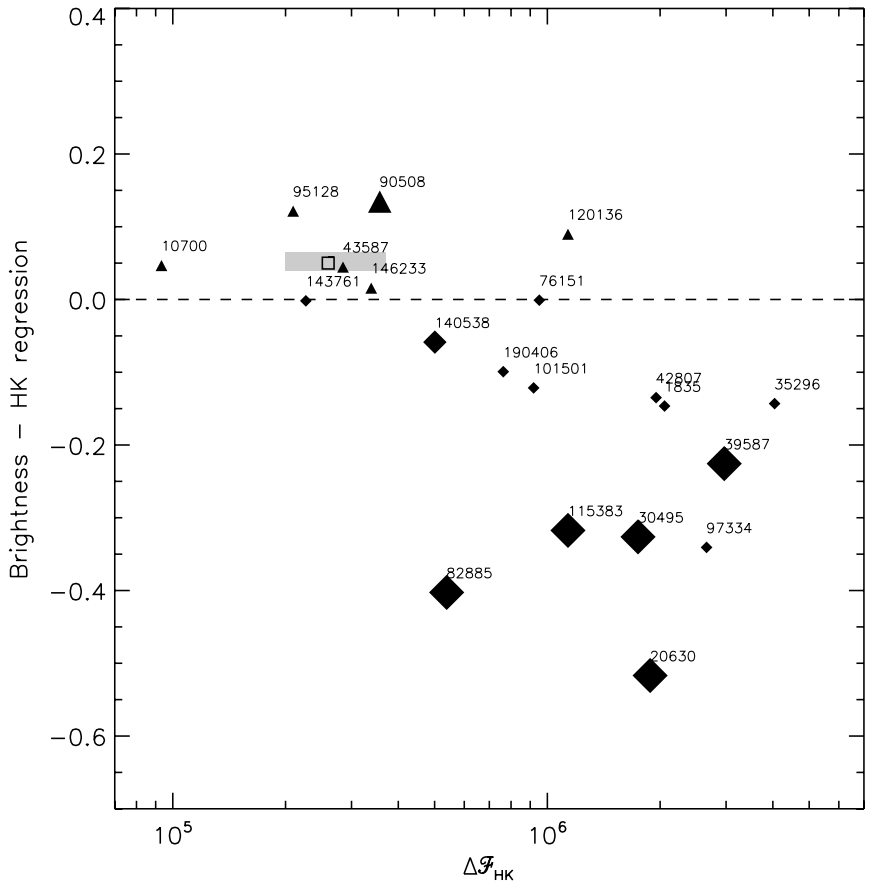

Figure 15. Direct vs. inverse activity correlations in all stars for which variability was detected. Stars above the dashed line show direct correlations, those below it, inverse correlations. The solar activity excursion is shown by the gray bar.

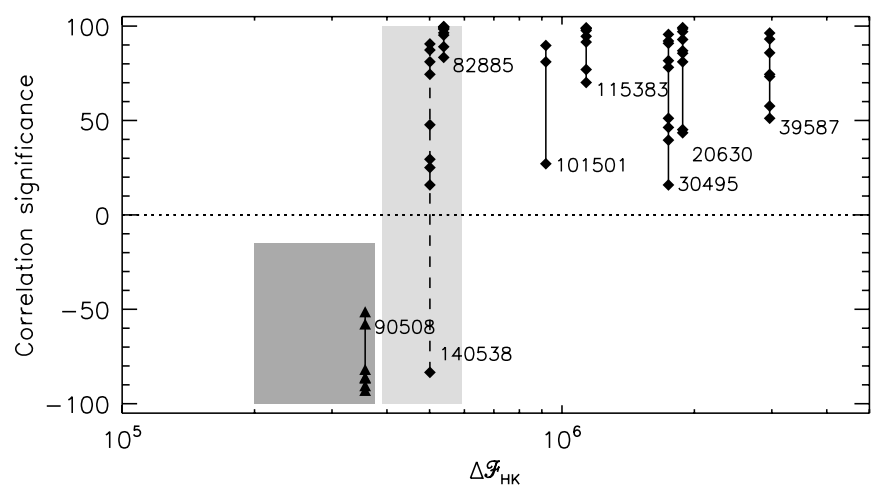

Figure 16. Activity-brightness correlations in four-year moving boxcars in all stars with significant overall correlations and detected photometric variations. Stars are plotted at their grand mean excess flux level. The dark gray bar shows the solar activity excursion, while the light gray bar shows the excursion of HD 140538; this star appears to change the sense of its behavior with activity.

prompted to investigate this by the interesting behavior of HD 140538 (Section 3.3), which appears to change the sense of its activity-brightness correlation on timescales of $4 \mathrm{yr}$, possibly in response to a transition from a noncycling to a cycling state. The criteria above whittle our sample down to eight stars, but for this dreadfully small group at least, there is a clear division of behavior. Active stars with $\Delta \mathcal{F}_{\mathrm{HK}}>5 \times 10^{5}$ always exhibit inverse correlations. The one inactive star with a moderately significant correlation, HD 90508, is always direct. Between these regimes lies HD 140538, which appears as the lone "crossover" star, exhibiting a wide range of correlations of timescales of 4 yr.

Such an inadequate sample cannot fully constrain the activity at which a star might flip from spot-dominated to faculaedominated brightness variations, but even this small sample demonstrates that these regimes are far from sharply defined. The most active Sun (whose excursion is shown by a dark gray bar) and 18 Sco both nearly overlap HD 140538's minima (light gray bar), so we should not be surprised that stars comparable to the present-day Sun might show weak or no activity-brightness correlations. Likewise, HD 82885, which exhibits the tightest inverse correlation of any of our targets, is not a highly active star, lying only slightly above HD 140538 in $\Delta \mathcal{F}_{\mathrm{HK}}$ (and, for that matter, $R_{\mathrm{HK}}^{\prime}$ ). Wherever the gradual transition from faculae-dominated to spot-dominated luminosity variation lies, it appears, on the basis of these eight stars at least, to be not far from the upper activity levels of modern solar cycles.

\section{CONCLUSIONS}

We have examined the chromospheric activity and photometric variations in 28 Sun-like stars over time periods ranging from 6 to $15 \mathrm{yr}$. The sample includes seven stars that overlap the targets discussed in Lockwood et al. (2007) and 21 stars that are the closest bright solar analogs. This sample allows us to draw the following conclusions.

1. We find little evidence for strong Sun-like activitybrightness correlations among the closest bright solar analogs. Several stars, including 18 Sco, exhibit levels of cycle-timescale photometric variation comparable to or less than the Sun's, so the Sun's photometric variability is not unusually low relative to its closest bright analogs.

2. The canonical activity index $R_{\mathrm{HK}}^{\prime}$ is not a good discriminant of Maunder Minimum candidate stars, or low-activity stars in general, at least not unless additional parameters such as metallicity are properly accounted for.

3. The excess magnetic fluxes, as inferred from $\Delta \mathcal{F}_{\mathrm{HK}}$ of the potential Maunder Minimum candidates HD 4307, 10700, 88986 , and 143761, are found to range from somewhat less than to comparable to the modern solar minimum.

4. The recent photometric behavior of the solar twin 18 Scorpii has been largely uncorrelated with its chromospheric activity. The overall activity level and cycle amplitude reported by Hall et al. (2007) is recovered and is very similar to the Sun's, but 18 Sco now exhibits at best a very weak activity-brightness correlation.

5. The star HD 140538 appears to have made a transition from a noncycling to a cycling state. In concert with that, there is evidence for a rather rapid change from direct activity-brightness variations in the low-activity state to inverse variations in the cycling state. Of the stars in our sample with significant activity-brightness correlations, HD 140538 is the only one that changes the sense of its correlation in any four-year subset of the time series. It therefore appears to define at least a part of the regime in which stars may change from faculae-dominated to spotdominated luminosity variations. This regime lies only slightly above the modern solar maximum.

The authors greatly appreciate the referee's exceptionally prompt report and useful comments on this paper. This work was supported by grant ATM-0742144 from the National Science Foundation. J.C.H., G.W.L., and B.A.S. thank the NSF divisions of Atmospheric Sciences (ATM) and Astronomical Sciences (AST) for support of the SSS program, and Program Director Paul Bellaire for thorough and careful help with our grant. G.W.H. acknowledges long-term support from NASA, NSF, Tennessee State University, and the State of Tennessee through its Centers of Excellence Program. S.H.S. was supported by NASA contract NNM07AB07C to SAO (Hinode XRT). Hinode is a Japanese mission developed and launched by ISAS/JAXA, with NAOJ as domestic partner and NASA and STFC (UK) 
as international partners. It is operated by these agencies in co-operation with ESA and the NSC (Norway). This work has made use of the SIMBAD astronomical database, operated at the CDS, Strasbourg, France.

\section{REFERENCES}

Baliunas, S. L., \& Jastrow, R. 1990, Nature, 348, 520

Baliunas, S. L., et al. 1995, ApJ, 438, 269

Bidelman, W. P. 1951, ApJ, 113, 304

Carney, B. W. 1978, AJ, 83, 1087

Cayrel de Strobel, G. 1989, A\&AR, 7, 243

Crawford, D. L., Barnes, J. V., Faure, B. Q., \& Golson, J. C. 1966, AJ, 71, 709

Duncan, D. K., et al. 1991, ApJS, 76, 383

Eddy, J. 1976, Science, 192, 1189

Fröhlich, C., \& Lean, J. 1998, Geophys. Res. Lett., 25, 4377

Gray, R. O., et al. 2003, AJ, 126, 2048

Hall, J. C., Henry, G. W., \& Lockwood, G. W. 2007, AJ, 133, 2206

Hall, J. C., \& Lockwood, G. W. 1995, ApJ, 438, 404

Hall, J. C., \& Lockwood, G. W. 2004, ApJ, 614, 942

Hall, J. C. Lockwood, G. W., \& Skiff, B. A. 2007, AJ, 133, 862

Hardorp, J. 1978, A\&A, 63, 383

Henry, G. W. 1999, PASP, 111, 845
Henry, T. J., Soderblom, D. R., Donahue, R. A., \& Baliunas, S. L. 1996, AJ, 111,439

Isobe, T., Feigelson, E. D., \& Nelson, P. I. 1986, ApJ, 306, 490

Johnson, H. L., Iriarte, B., Mitchell, R. I., \& Wisniewksi, W. Z. 1966, Commun. Lunar. Planet Lab., 4, 99

Judge, P. G., \& Saar, S. H. 2007, ApJ, 663, 643

Lockwood, G. W., et al. 2007, ApJS, 171, 260

Melendez, J., \& Ramirez, I. 2007, ApJ, 669, L89

Mermilliod, J.-C. 1986, Catalog of Eggen's UBV Data (Strasbourg: CDS)

Olsen, E. H. 1993, A\&AS, 102, 89

Olsen, E. H. 1994, A\&AS, 104, 429

Porto de Mello, G. F., \& da Silva, L. 1997, ApJ, 482, L89

Radick, R. R., Lockwood, G. W., Skiff, B. A., \& Baliunas, S. L. 1998, ApJS, 118,239

Rutten, R. G. M., \& Schrijver, C. J. 1987, A\&A, 177, 155

Saar, S. H. 2006, BAAS, 38, 240

Schröder, C., Reiners, A., \& Schmitt, J. H. M. M. 2009, A\&A, 493, 1107

Skiff, B. A., \& Lockwood, G. W. 1986, PASP, 98, 338

Soubiran, C., \& Triaud, A. 2004, A\&A, 418, 1089

Valenti, J. A., \& Fischer, D. A. 2005, ApJS, 159, 141

Wang, Y.-M., Lean, J. L., \& Sheeley, N. R., Jr. 2005, ApJ, 625, 522

Willson, R. C. 1997, Science, 277, 1963

Wilson, O. C. 1978, ApJ, 153, 221

Wright, J. T. 2004, AJ, 128, 1273 